\title{
Bestimmung von Transferfaktoren von Caesium in der Kette Boden-Rapspflanze-Rapsblüte-Rapshonig
}

\author{
D. Molzahn 1, A. Klepsch 2, U. Assmann-Wertmüller 2 \\ 1 Kernchemie im Fachbereich Physikalische Chemie, Philipps-Universität Marburg; \\ ${ }^{2}$ Hessische Landesanstalt für Tierzucht - Abteilung für Bienenzucht, 3575 Kirchhain, GFR
}

(Eingegangen 2. September 1988; angenommen 28. Juli 1989)

Zuzammenfassung - In dieser Arbeit wird der Übergang von radioaktivem Caesium aus dem Boden in die Rapspflanzen (Brassica napus var. oleifera), die Rapsblüten und den Rapshonig untersucht. Durch Messung der Gamma-Aktivität des Caesiums mit einem Germanium-Detektor bei Meßzeiten von 15 bis 30 Stunden je Probe sind folgende Transferfaktoren ermittelt worden : Kette Erde-Rapspflanze $f(C s)=0,116 \pm 0,080$, Kette Erde-Rapsblüten $f(C s): 0,065 \pm 0,075$ und Kette Erde-Rapshonig $f(C s)=0,098 \pm 0,044$. Die Schwankungsbreite der einzelnen Meßergebnisse ist relativ groß, verursacht durch eine Reihe schwer zugänglicher Umwelteinflüsse. Trotzdem liegen die Mittelwerte im Rahmen der in der Literatur aufgeführten Grenzen. Die gefundenen niederen Transferfaktoren und die geringe Belastung des Rapshonigs mit radioaktivem Caesium bedeuten, daß der Verzehr von Rapshonig keine gesundheitliche Gefährdung des Verbrauchers verursacht.

Boden - Rapspflanze - Rapsblüte - Rapshonig - Caeșium

\section{EINLEITUNG}

Eine Folge der ersten Atombombenexplosionen im Jahre 1945, der oberirdischen Kernwaffenversuche bis 1962 und des Unfalls im Kernkraftwerk Tschernobyl am 26. April 1986 ist die Belastung der Umwelt mit radioaktiven Spaltprodukten. Es besteht die Möglichkeit, daß diese Spaltprodukte von Pflanzen mit ihrer Nahrung aus dem Boden aufgenommen werden und da $\beta$ somit ein Transfer radioaktiver Nuklide aus dem Boden auch in Nahrungsmittel erfolgt. Infrage dafür kommen die beiden Caesium-Isotope ${ }^{134} \mathrm{Cs}$ und ${ }^{137} \mathrm{Cs}$ mit Halbwertszeiten von $t_{1 / 2}=2,04$ Jahren und $t_{1 / 2}$
$=30,16$ Jahren sowie das StrontiumIsotop ${ }^{90} \mathrm{Sr}\left(t_{1 / 2}=28,6\right.$ Jahre $)$. Alle anderen Spaltprodukte spielen wegen ihrer relativ kurzen Halbwertszeiten praktisch keine Rolle. Die Caesium-Isotope lassen sich mit der Methode der Gammaspektrometrie relativ einfach nachweisen, eine vorherige Bearbeitung der Proben entfällt im allgemeinen. Im Gegensatz dazu steht das ${ }^{90} \mathrm{Sr}$, ein reiner Betastrahler, zu dessen Bestimmung eine umfangreiche und langwierige Probenvorbereitung vonnöten ist. Mit der hier beschriebenen Methode ist ${ }^{90} \mathrm{Sr}$ nicht nachweisbar. Ziel dieser Arbeit war es, den Übergang von Caesium aus dem Boden über die Pflanzen in ein Nahrungsmittelendprodukt zu verfolgen, spe- 
zieller gesagt, den Transferfaktor für ${ }^{137} \mathrm{Cs}$ aus dem Boden über die Rapspflanze in den Rapshonig zu bestimmen. Der Rapshonig wurde ausgewählt, weil in diesem Fall dem Honig bestimmter Bienenvölker auch bestimmte Rapsfelder zugeordnet werden können. Bei den später gewonnenen Honigsorten ist dagegen die Vielfalt der als Nektarquelle infrage kommenden Pflanzen zu groß.

Die Untersuchungen erfolgten mit Produkten des Jahres 1987. Das bedeutet, daß echte Faktoren für den Übergang des Caesiums aus dem Boden über die Pflanze in den Honig bestimmt werden konnten. Messungen dieser Art wären dagegen im Jahre 1986 nicht durchführbar gewesen, da damals nach dem Reaktorunfall die Pflanzen durch den Regen in beträchtlichem Maße oberflächlich kontaminiert waren und ein Caesiumtransfer Boden-Pflanze noch nicht stattgefunden hatte. Im Jahre 1987 enthielt die Luft indessen praktisch keine künstlichen Radioisotope mehr. Daher erfolgte auch keine Kontamination der Pflanzen an der Oberfläche. Festgestellte Cs-Aktivitäten in den Pflanzen sind also auf reinen Transfer dieses Elements aus dem Boden zurückzuführen.

Über Kontaminationsmessungen an Honig der Ernte 1986 haben verschiedene Autoren ausführlich berichtet (Horn et al., 1986; Kaatz, 1986; Klepsch et al., 1987; Dustmann et al., 1988). Veröffentlichungen über den Transfer von Caesium aus dem Boden in den Honig sind uns dagegen noch nicht bekannt. Auch Ford et al. (1988), die den Caesium-Gehalt in Bienen untersuchen, gehen auf den Transfer des Caesiums von der Biene in den Honig nicht ein.

Als Transferfaktor $f_{e}$ für ein radioaktives Element e bezeichnet man das Verhältnis: $f_{e}=$ spezifische Konzentration des Radionuklides e in der Pflanze / spezifische Konzentration des Radionuklides $e$ in der Erde.

Dabei bezieht sich die Angabe der spezifischen Konzentration eines Radionuklides in der Erde auf die Trockenmasse, in Lebens- und Futtermitteln dagegen auf deren Feuchtmasse, eine Regelung, die indessen nicht einheitlich ist. In dieser Arbeit wurde für die Erde die Trockenmasse, für die Pflanzen und den Honig die Feuchtmasse verwendet.

Allgemein liegen die Transferfaktoren für Grünpflanzen, ermittelt über Lysimeterversuche, im Bereich zwischen 0,00003 (Getreide) und 6,0 (Klee), bezogen allerdings auf Trockenmasse (Bundesminister des Inneren, 1981; Aurand et al., 1982; Steffens et al., 1979/80 und 1986). Für Raps gibt Frederiksson (1966) ebenfalls auf Trockenmasse bezogene Transferfaktoren zwischen 0,1 (auf Lehmboden aus Schweden) und 13,5 (auf Laterit aus Brasilien) an.

\section{PROBENNAHME UND MESSMETHODE}

\section{StandortauswahI}

Im Jahre 1986 hatten Aktivitätsmessungen (Molzahn, 1987; Wiesmüller, 1987) für den Südosten des Landkreises Marburg-Biedenkopf relativ hohe Caesium-Belastungen ergeben (Flächenbelastung bis zu $7500 \mathrm{~Bq} / \mathrm{m}^{2}$ ). Drei Felder in der Nähe dieses Gebietes, nämlich bei Wäldershausen, Rauischholzhausen und am Flugplatz Neu-Ulrichstein erschienen daher für die geplanten Untersuchungen geeignet. 


\section{Probennahme}

Die Einsammlung der Boden- und Rapspilanzenproben erfolgte am 12. Mai 1987, die der Rapsblüten am 27. Mai 1987 (spätere Blüte). Auf den Feldern bei Wäldershausen und Rauischholzhausen wurden an jeweils 4 Stellen, auf dem Feld am Flugplatz an 2 Stellen Proben genommen. Die Entfernung zwischen den Probennahmestellen eines Feldes betrug jeweils zwischen $100 \mathrm{~m}$ und $150 \mathrm{~m}$. Bei einer geschätzten Umbruchtiefe von $25 \mathrm{~cm}$ und einer damaligen Reichweite der Rapspflanzenwurzeln bis ca. $20 \mathrm{~cm}$ in den Erdboden genügte die Entnahme von Erdproben bis zu $30 \mathrm{~cm}$ Tiefe, aufgeteilt in Teilprofile $0-10 \mathrm{~cm}, 10-20 \mathrm{~cm}$ und $20-$ $30 \mathrm{~cm}$. Bei allen drei Standorten handelt es sich dabei um dem Bodentyp Parabraunerde.

Nach der Rapstracht wurden die von den Völkern der untersuchten Standorte gesammelten Honige abgeschleudert. Pro Schleuderung wurde jeweils eine Honigprobe entnommen und auf ihren Caesiumgehalt hin untersucht. Insgesamt ergaben sich in Wäldershausen am 26.6.1987 elf Honigproben von 24 Völkern, in Rauischholzhausen am 29.6.1987 neun Honigproben von 27 Völkern und in Neu-Ulrichstein Flugplatz am 2.7.1987 vier Honigproben von 8 Völkern. In Wäldershausen konnten $386,6 \mathrm{~kg}$ Honig, in Rauischholzhausen $409,9 \mathrm{~kg}$ und in Neu-Ulrichstein Flugplatz $135,4 \mathrm{~kg}$ geerntet werden.

Wie über Pollenanalyse nachgewiesen wurde, handelt es sich bei allen geernteten Honigen um Rapshonig mit einem Rapsanteil von über $95 \%$.

\section{Probenvorbereitung}

Die Erdproben wurden bei $100{ }^{\circ} \mathrm{C}$ im Trockenschrank getrocknet, alle anderen Proben blieben dagegen unbearbeitet. Die Messung der Erd-, Rapspflanzen- und Rapsblütenproben erfolgte in $250 \mathrm{ml}$ Plastikbechern, die der Honigproben in $250 \mathrm{ml}$ Honiggläsern. Für dieses Volumen war der Detektor quantitativ geeicht.

\section{Meßtechnik}

Die Caesiumisotope ${ }^{134} \mathrm{Cs}$ und ${ }^{137} \mathrm{Cs}$ können durch ihre charakteristische Gammastrahlung nachgewiesen werden $(E \gamma=604,7 \mathrm{keV}$ für ${ }^{134} \mathrm{Cs}$, $\mathrm{E} \gamma=661,6 \mathrm{keV}$ für $\left.{ }^{137} \mathrm{Cs}\right)$. Zur Messung dieser Gammastrahlung wurden GermaniumDetektoren der Firmen Ortec bzw. Princeton GammaTech verwendet. Die Aufnahme der Spektren erfolgte mit einem Vielkanalanalysator ND 66 der Fa. Nuclear Data, die Auswertung der Spektren mit einem Tandom Personal Computer über die Programme MEDIGAM bzw. GAMMAW (Westmeier, 1986). Die Detektoren waren gegen die Umgebungsstrahlung durch Bleiburgen von $10 \mathrm{~cm}$ bzw. $5 \mathrm{~cm}$ Wandstärke allseitig abgeschirmt. $\mathrm{Da}$ die Detektoren auf Strahlung verschiedener Energie unterschiedlich reagieren und außerdem nur einem geringen Bruchteil der gleichmäßig in alle Richtungen ausgehenden Strahlung erfassen können, mußte ihre Nachweiswahrscheinlichkeit für die in dieser Arbeit generell verwendeten $250 \mathrm{ml}$ Volumenproben mit Hilfe geeichter Gammastan-

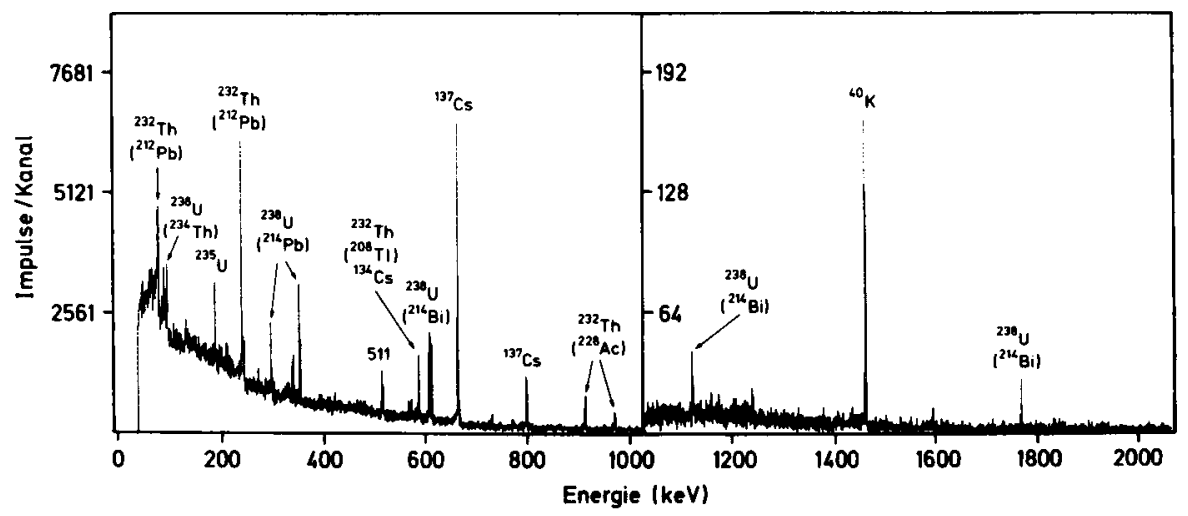

Abb. 1. Gammaspektrum einer Erdprobe. 
dards ermittelt werden (Jäckel et al., 1987). Die Meßzeiten lagen in der Regel zwischen 15 und 30 Stunden. Dafür errechnen sich Nachweisgrenzen für beide Isotope zwischen $0,19 \mathrm{~Bq}$ und $0,14 \mathrm{~Bq}$. Abb. 1 zeigt das Gammaspektrum einer Meßprobe.

\section{ERGEBNISSE}

\section{Erdproben}

Aus der Literatur (z.B. Frederiksson et al., 1966) und eigenen Messungen (Molzahn et al., 1989) ist bekannt, daß die Eindring- geschwindigkeit des Caesiums in unbearbeiteten Boden gering ist. So ließen sich im Herbst 1987 noch annähernd $80 \%$ der Gesamtcaesium-Aktivität aus Tschernobyl in den obersten $6 \mathrm{~cm}$ Bodenschicht nachweisen. Bei bearbeitetem Boden erfolgte dagegen für die oberen $20-30 \mathrm{~cm}$ Schichtdicke durch das Umpflügen bzw. Umgraben eine Durchmischung. Es war daher nicht verwunderlich, daß die Erdproben aus den 3 verschiedenen Schichten ähnliche Caesium-Belastungen aufwiesen. Die in Tabelle I aufgeführten Aktivitätswerte für die verschiedenen Standorte geben den Mittelwert der Belastung aus jeweils 3 Messungen für die gesamte Tiefe an. Die Gesamtcaesium-Aktivität der Erdproben liegt zwischen 12,4 Bq/kg und 18,7 Bq/kg Trockengewicht (Mittelwert je Standort).

Tabelle I. Aktivitätsgehalt in Erdproben. Tag der Probennahme 12. Mai 1987, die Messung der Proben erfolgte von Mai-Juli 1987.

Aktivität in Bq/kg Trockengewicht

\begin{tabular}{lcrrrrr}
\multicolumn{1}{c}{ Standort } & Probe & ${ }^{134} \mathrm{Cs}$ & ${ }^{137} \mathrm{Cs}$ & ${ }^{232} \mathrm{Th}$ & ${ }^{238} \mathrm{U}$ & ${ }^{40} \mathrm{~K}$ \\
\hline Wäldershausen & I & $3,9 \pm 0,2$ & $14,1 \pm 0,4$ & $30 \pm 1$ & $23 \pm 1$ & $345 \pm 13$ \\
& II & $3,4 \pm 0,3$ & $10,7 \pm 0,4$ & $27 \pm 1$ & $21 \pm 1$ & $342 \pm 10$ \\
& III & $2,4 \pm 0,2$ & $7,5 \pm 0,2$ & $15 \pm 1$ & $13 \pm 1$ & $209 \pm 7$ \\
& & $1,6 \pm 0,2$ & $6,2 \pm 0,3$ & $17 \pm 1$ & $15 \pm 1$ & $270 \pm 7$ \\
Mittelwert & & & & & & \\
Wäldershausen & & $2,8 \pm 0,3$ & $9,6 \pm 0,4$ & $22 \pm 1$ & $25 \pm 1$ & $292 \pm 11$ \\
Flugplatz & I & $5,9 \pm 0,2$ & $19,5 \pm 0,4$ & $34 \pm 1$ & $25 \pm 1$ & $495 \pm 9$ \\
& II & $2,0 \pm 0,2$ & $9,9 \pm 0,4$ & $27 \pm 1$ & $21 \pm 1$ & $408 \pm 11$ \\
Mittelwert & & & & & & \\
Flugplatz & & $4,0 \pm 0,3$ & $14,7 \pm 0,6$ & $31 \pm 1$ & $23 \pm 1$ & $452 \pm 14$ \\
Rauischholzhausen & II & $1,6 \pm 0,2$ & $10,3 \pm 0,5$ & $28 \pm 1$ & $15 \pm 1$ & $313 \pm 11$ \\
& II & $5,6 \pm 0,4$ & $20,3 \pm 0,8$ & $23 \pm 1$ & $15 \pm 1$ & $292 \pm 15$ \\
& III & $2,5 \pm 0,3$ & $10,2 \pm 0,5$ & $31 \pm 1$ & $22 \pm 1$ & $424 \pm 9$ \\
& & $1,6 \pm 0,2$ & $6,2 \pm 0,3$ & $33 \pm 1$ & $20 \pm 1$ & $349 \pm 11$ \\
Mittelwert & & & & & & \\
Rauischholzhausen & & $2,8 \pm 0,3$ & $11,8 \pm 0,6$ & $29 \pm 1$ & $18 \pm 1$ & $345 \pm 1$ \\
\hline
\end{tabular}


Neben den Caesium-Meßwerten sind in Tabelle I auch die der natürlichen radioaktiven Bestandteile des Erdbodens angegeben. Diese findet man praktisch in allen Böden. Kalium ist an den oberen Schichten der Erdoberfläche zu etwa 2,5 Gewichtsprozent beteiligt, davon entfallen $0,0117 \%$ auf das radioaktive Nuklid ${ }^{40} \mathrm{~K}$. Ein Kubikmeter Erde enthält etwa $3 \mathrm{~g}$ Natururan (238 $\mathrm{U})$ und $3 \mathrm{~g}$ Naturthorium (232Th) sowie deren Zerfallsprodukte. Da viele Düngemittel Kalium, Uran und Thorium enthalten, ist eine zusätzliche Anreicherung auf entsprechend gedüngten Feldern möglich. Die Aktivitäten dieser natürlichen radioaktiven Nuklide übersteigen die der Caesium-Isotope teilweise deutlich, besonders im Falle des
40K. Die Uranaktivität wurde über das Zerfallsprodukt ${ }^{214} \mathrm{Bi}$ mit der Gammaenergie $609,3 \mathrm{keV}$, die Thoriumaktivität über das ${ }^{212} \mathrm{~Pb}$ mit $\mathrm{E}=238,6 \mathrm{keV}$ und die Kaliumaktivität über die Gammaenergie $E=1460,8$ keV des ${ }^{40} \mathrm{~K}$ ermittelt.

Wie die Tabelle I zeigt, variieren die Aktivitätswerte der einzelnen Probennahmestellen eines Feldes zum Teil beträchtlich.

\section{Rapspflanzen und Rapsblüten}

In der Tabelle II sind neben den Mittelwerten der Gesamtcaesiumaktivität der Erdproben die Gesamtcaesium-Aktivitätswerte

Tabelle II. Gesamtcaesiumgehalt in Erdproben, Rapspilanzen und Rapsblüten. Die Sammlung der Erdproben und Rapspflanzen erfolgte am 12. Mai 1987, die der Rapsblüten am 22. Mai 1987. Die Messungen wurden in Mai (Erde und Rapspflanzen) bzw. Ende Mai/Anfang Juni (Rapsblüten) durchgeführt.

\begin{tabular}{lcccc}
\hline & \multicolumn{4}{c}{ Gesamtcaesiumaktivität in Bq/kg } \\
Standort & Probe & $\begin{array}{c}\text { Erdproben } \\
\text { (Trockenmasse) }\end{array}$ & $\begin{array}{l}\text { Rapspflanzen } \\
\text { (Feuchtmasse) }\end{array}$ & $\begin{array}{l}\text { Rapsblüten } \\
\text { (Feuchtmasse) }\end{array}$ \\
\hline Wäldershausen & I & $18,0 \pm 0,4$ & $1,6 \pm 0,8$ & $2,4 \pm 0,8$ \\
& II & $14,1 \pm 0,5$ & $2,4 \pm 1,5$ & $0,5 \pm 0,5$ \\
& III & $9,9 \pm 0,3$ & $3,0 \pm 1,0$ & $0,9 \pm 0,6$ \\
& IV & $7,8 \pm 0,7$ & $2,1 \pm 0,7$ & $0,8 \pm 1,1$ \\
Mittelwert & & $12,5 \pm 0,5$ & $2,3 \pm 1,2$ & $1,2 \pm 0,9$ \\
Wäldershausen & & & & \\
Flugplatz & I & $25,4 \pm 0,4$ & $0,7 \pm 0,7$ & $1,6 \pm 0,7$ \\
& II & $11,9 \pm 0,4$ & $0,5 \pm 0,9$ & $1,1 \pm 0,7$ \\
Mittelwert & & $18,7 \pm 0,6$ & $0,6 \pm 1,1$ & $1,4 \pm 1,0$ \\
Flugplatz & & & & \\
Rauischholzhausen & I & $11,9 \pm 0,5$ & $1,7 \pm 1,0$ & $0,5 \pm 0,6$ \\
& II & $25,9 \pm 0,9$ & $1,2 \pm 0,7$ & $0,4 \pm 0,5$ \\
& III & $12,8 \pm 0,6$ & $1,2 \pm 0,7$ & $0,3 \pm 0,7$ \\
& IV & $7,8 \pm 0,4$ & $1,9 \pm 0,7$ & $0,4 \pm 0,9$ \\
Mattelwert & & $14,6 \pm 0,7$ & $1,5 \pm 0,9$ & $0,4 \pm 0,8$ \\
Rauischholzhausen & & & & \\
\hline
\end{tabular}


für Rapspflanzen und Rapsblüten aufgeführt. Die Rapspflanzen wurden im Mai 1987, die Rapsblüten Ende Mai-Anfang Juni 1987 gemessen. Die Meßwerte liegen sowohl für die Rapspflanzen als auch für die Rapsblüten im Bereich von 0,3 bis 3,0 $\mathrm{Bq} / \mathrm{kg}$. Im Mittel ist die Caesium-Aktivität der Rapsblüten jedoch kleiner als die der Rapspflanzen.

Im Gegensatz zu den Meßergebnissen an den Erdproben ist die Caesium-Aktivität bei den Rapsproben am Standort Wäldershausen höher als die am Standort Flugplatz. Jedoch ist zu beachten, daß wir nur einige wenige Meßpunkte haben, die allesamt kleine Zahlen mit relativ hoher Meßunsicherheit ergeben. Innerhalb der Fehlergrenze ist durchaus eine Übereinstimmung der Meßwerte festzustellen.

\section{Honig}

Die Meßwerte der mittleren Caesiumaktivität in den Honigproben der 3 Standorte sind in Tabelle III aufgetragen. Die "höchste" Belastung des Honigs mit Caesium finden wir in den Honigen des Standortes Flugplatz. Dieses Ergebnis stimmt zwar mit dem der Erdproben überein, nicht aber mit dem der Rapspflanzen und
Rapsblüten. Die Caesium-Werte in den Honigen der beiden anderen Standorte liegen sehr niedrig, aber noch über der Nachweisgrenze unserer Methode.

Zum Vergleich sei noch die im hiesigen Rapshonig der Vorjahre gemessene Gesamtcaesium-Aktivität aufgeführt. Im Monat Mai 1986 lag der Mittelwert aus 4 Proben Rapshonig bei $15 \mathrm{~Bq} / \mathrm{kg}$, im Monat Juni 1986 bei $18 \mathrm{~Bq} / \mathrm{kg}$ (aus 54 Proben). Der Höchstwert einer Probe betrug 118 $\mathrm{Bq} / \mathrm{kg}$. Dagegen ergab eine Überprüfung von im Jahre 1985 geschleuderten Rapshonigen bei allen 35 untersuchten Proben keine Kontamination mit radioaktiven Caesium-Isotopen (Assmann-Wertmüller et al., 1989).

\section{Transferfaktoren für Caesium}

Ziel der Untersuchung war die Ermittlung der Faktoren für den Caesiumtransfer

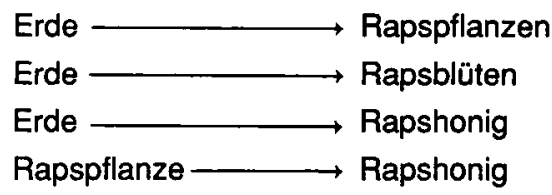

bezogen auf den Gesamtcaesiumgehalt. In der Tabelle IV sind die Faktoren für die Übergänge Erde $\longrightarrow$ Rapspflanzen und Erde $\rightarrow$ Rapsblüten zunächst für die einzel-

Tabelle III. Caesium-Aktivität in Rapshonig vom Frühjarhr 1987. Schleuderung Juni-Juli 1987, Messung im Juli 1987.

\begin{tabular}{lcc}
\hline Standort & $\begin{array}{c}\text { Zahl der gemessenen } \\
\text { Proben }\end{array}$ & $\begin{array}{c}\text { Mittlere Gesamt- } \\
\text { caesium-Aktivität } \\
\text { in Bq/kg }\end{array}$ \\
\hline Wäldershausen & 11 & $0,5 \pm 0,2$ \\
Flugplatz & 4 & $3,3 \pm 1,0$ \\
Rauischholzhausen & 9 & $0,6 \pm 0,2$ \\
\hline
\end{tabular}


Tabelle IV. Transferfaktoren für Caesium für die Pfade Erde - Rapsblüten, Erde - Rapshonig und Rapspflanze - Rapshonig.

\begin{tabular}{|c|c|c|c|c|c|}
\hline \multirow[t]{2}{*}{ Standort } & \multicolumn{5}{|c|}{ Transferfaktoren $f(C s)$} \\
\hline & & $\begin{array}{c}\text { Erde- } \\
\text { Rapspflanze }\end{array}$ & $\begin{array}{c}\text { Erdo- } \\
\text { Rapsblüten }\end{array}$ & $\begin{array}{c}\text { Erdo- } \\
\text { Rapshonig }\end{array}$ & $\begin{array}{l}\text { Rapspflanze- } \\
\text { Rapshonig }\end{array}$ \\
\hline Wäldershausen & $\begin{array}{l}\text { I } \\
\text { II } \\
\text { III } \\
\text { IV }\end{array}$ & $\begin{array}{l}0,089 \pm 0,044 \\
0,170 \pm 0,107 \\
0,303 \pm 0,091 \\
0,269 \pm 0,091\end{array}$ & $\begin{array}{l}0,133 \pm 0,045 \\
0,035 \pm 0,035 \\
0,103 \pm 0,0141 \\
0,103 \pm 0,0141\end{array}$ & & \\
\hline $\begin{array}{l}\text { Mittelwert } \\
\text { Wäldershausen }\end{array}$ & & $0,208 \pm 0,013$ & $0,091 \pm 0,095$ & $0,040 \pm 0,016$ & $0,217 \pm 0,143$ \\
\hline Flugplatz & II & $\begin{array}{l}0,028 \pm 0,028 \\
0,042 \pm 0,075\end{array}$ & $\begin{array}{l}0,063 \pm 0,028 \\
0,092 \pm 0,059\end{array}$ & & \\
\hline $\begin{array}{l}\text { Mittelwert } \\
\text { Flugplatz }\end{array}$ & & $0,035 \pm 0,080$ & $0,078 \pm 0,065$ & $0,176 \pm 0,054$ & $5,50 \pm 10,22$ \\
\hline Rauischholzhausen & $\begin{array}{l}\text { I } \\
\text { II } \\
\text { III } \\
\text { IV }\end{array}$ & $\begin{array}{l}0,143 \pm 0,084 \\
0,046 \pm 0,027 \\
0,094 \pm 0,055 \\
0,24 \pm 0,091\end{array}$ & $\begin{array}{l}0,042 \pm 0,050 \\
0,015 \pm 0,019 \\
0,023 \pm 0,055 \\
0,051 \pm 0,115\end{array}$ & & \\
\hline $\begin{array}{l}\text { Mittelwert } \\
\text { Rauischholzhausen }\end{array}$ & & $0,132 \pm 0,084$ & $0,042 \pm 0,050$ & & \\
\hline $\begin{array}{l}\text { Mittelwert aus allen } \\
\text { Einzelproben }\end{array}$ & & $0,116 \pm 0,080$ & $0,065 \pm 0,075$ & $0,098 \pm 0,044$ & $2,039 \pm 7,230$ \\
\hline
\end{tabular}

nen Probenahmestellen eines jeden Feldes zusammengestellt, sodann daraus der Mittelwert für den Gesamtstandort. Die Faktoren für die Übergänge Erde Rapshonig und Rapspflanze Rapshonig konnten dagegen nur aus dem Mittelwert der Belastung von Erde bzw. Rapsflanze gegenüber des Rapshonig ermittelt werden, da eine Zuordnung des Honigs zu einzelnen Probenahmestellen nicht möglich war. SchlieBlich wird der Mittelwert aus allen Einzelmessungen aufgeführt.

Im einzelnen ergibt sich :
- für die Kette Erde-Rapspflanze ein Mittelwert von $f(C s)=0,116 \pm 0,080$ bei einem Schwankungsbereich von 0,028 bis 0,303 ;

- für die Kette Erde-Rapsblüten ein Mittelwert von $f(C s)=0,065 \pm 0,075$ bei einem Schwankungsbereich von 0,015 bis 0,133 ; — für die Kette Erde-Rapshonig ein Mittelwert von $f(C s)=0,098 \pm 0,044$ bei einem Schwankungsbereich von 0,040 bis 0,176 ;

- für den Pfad Rapspflanze-Rapshonig ein Mittelwert von $f(C s)=2,039 \pm 7,230$ bei einem Schwankungsbereich von 0,217 bis 5,50 . 


\section{DISKUSSION}

In Tabelle $V$ sind die in dieser Arbeit für die Kette Boden-Pflanze ermittelten Transferfaktoren den aus der Literatur entnehmbaren Werten gegenübergestellt. Schwierigkeiten bereitet, da $B$ manche Werte auf Feuchtsubstanz Pflanze, andere auf Trockensubstanz Pflanze bezogen sind. Übersichtsmessungen haben ergeben, daß der Trockenanteil bei etwa 12,5\% der Feuchtmasse liegt. Berücksichtigt man diesen Wert, so liegen die hier bestimmten Transferfaktoren für die Kette BodenRapspflanze/Rapsblüte zwischen 0,12 und 2,42 . Der Minimalwert liegt damit deutlich höher als vergleichbare Literaturwerte, der Maximalwert jedoch unterhalb mancher von anderen Autoren angegebenen Werte.
Zu beachten ist, daß die Mehrzahl der Vergleichswerte über Lysimeterversuche ermittelt wurde, die Werte dieser Arbeit aber aus der unbeeinflußten Natur stammen. Aus allen Werten ist jedoch klar zu entnehmen, daß der Übergang des radioaktiven Caesiums aus dem Boden in die hier untersuchten Nutzpflanzen äußerst gering ist und keine Gefährdung der daraus erzeugten Lebensmittel hervorruft. $\mathrm{Da} B$ dies nicht auf alle Pflanzen zutrifft, beweisen z.B. die teilweise sehr hohen Transferfaktoren für einige Pilzarten.

Die oben getroffene Feststellung der geringen Gefährdung gilt auch für den Rapshonig. Ergänzend soll jedoch erwähnt werden, daß die bei Waldhonigen und besonders Heidehonigen auftretenden relativ hohen Caesium-Kontaminationen nicht auf einen Übergang des Caesiums vom Boden

Tabelle V. Übersicht über Transferfaktoren $\mathrm{f}(\mathrm{Cs})$ für die kette Boden-Nutzpflanzen.

\begin{tabular}{|c|c|c|c|c|}
\hline Pflanzenart & $\begin{array}{l}\text { Transfer- } \\
\text { faktor }\end{array}$ & $\begin{array}{l}\text { kenmasse (TM) } \\
\text { chtmasse (FM) }\end{array}$ & Bodenart & Autor \\
\hline Raps & $\begin{array}{r}0,1 \\
13,5\end{array}$ & $\begin{array}{l}\text { TM } \\
\text { TM }\end{array}$ & $\begin{array}{l}\text { Lehmboden } \\
\text { Laterit }\end{array}$ & $\begin{array}{l}\text { Frederiksson } \\
\text { (1966) }\end{array}$ \\
\hline Bodenbewuchs & $\begin{array}{l}0,037-0,13 \\
0,095-0,17\end{array}$ & $\begin{array}{l}F M \\
F M\end{array}$ & $\begin{array}{l}\text { Geest } \\
\text { Hochmoor }\end{array}$ & Heine (1978) \\
\hline Bodenbewuchs & 0,06 & FM & Mittelwert & Heine (1979) \\
\hline $\begin{array}{l}\text { Weidevegetation } \\
\text { Klee }\end{array}$ & $\begin{array}{l}0,0007-4,6 \\
0,04-6,0\end{array}$ & $\begin{array}{l}\text { TM } \\
\text { TM }\end{array}$ & & Biesold (1981) \\
\hline $\begin{array}{l}\text { Gras } \\
\text { Luzerne }\end{array}$ & $\begin{array}{ll}0,05 & -0,012 \\
0,002 & -0,010\end{array}$ & $\begin{array}{l}\text { TM } \\
\text { TM }\end{array}$ & $\begin{array}{l}\text { Parabraun } \\
\text { Parabraun }\end{array}$ & Biesold (1982) \\
\hline $\begin{array}{l}\text { Getreide } \\
\text { Blattgemüse }\end{array}$ & $\begin{array}{l}0,0014 \\
0,0062\end{array}$ & $\begin{array}{l}F M \\
F M\end{array}$ & $\begin{array}{l}\text { Parabraun } \\
\text { Parabraun }\end{array}$ & Steffens (1986) \\
\hline $\begin{array}{l}\text { Rapspflanze } \\
\text { Rapsblüte }\end{array}$ & $\begin{array}{l}0,028-0,303 \\
0,015-0,13\end{array}$ & $\begin{array}{l}F M \\
F M\end{array}$ & $\begin{array}{l}\text { Parabraun } \\
\text { Parabraun }\end{array}$ & diese Arbeit \\
\hline
\end{tabular}


in die Pflanze zurückzuführen sind, sondern daß sich in diesen Fällen noch vom ursprünglichen stark kontaminerten Regen her radioaktives Caesium z.B. auf den Nadeln bzw. dem Heidekraut befindet und direkt von den Bienen aufgenommen wird.

Aus Tabelle IV ist zu entnehmen, daß die einzelnen Werte für einen Pfad oft erheblich schwanken. Diese Schwankungen können viele, zum Teil schwer faßbare Ursachen haben. Zum einen ist nicht bekannt, wieviel des im Boden befindlichen Caesiums wirklich pflanzenverfügbar ist. Caesium verhält sich chemisch ähnlich dem Kalium. So kann zum anderen ein höherer verfügbarer Kaliumanteil im Boden eine geringere Aufnahme von Caesium in die Pflanze bewirken. Weiter ist die Caesium-Aktivität im Boden nicht konstant. Es gibt Orte, an denen das Oberflächenwasser zusammengelaufen ist und an denen sich daher auch eine erhöhte Caesium-Konzentration vorfinden mag. Das ein- oder zweimalige Umpflügen des Bodens nach dem Unfall von Tschernobyl wird noch nicht für eine vollkommen gleichmäßige Durchmischung gesorgt haben, daher kann manchen Pflanzenwurzeln ein größere Menge Caesium zur Verfügung stehen als anderen. Außerdem spielen pH-Wert, Ton- und Humusgehalt sowie eine Reihe weiterer Faktoren eine Rolle, die aber in dieser Arbeit nicht berücksichtigt werden konnten.

\section{DANK}

Für tatkräftige Hilfe beim Sammeln der Proben möchten wir den Herren Bernd Jäckel und Wilhelm Werner Danken, ebenso auch Herrn Peter Kocksholt für Mithilfe beim Messen der Proben sowie Frau Sabine Haase und Frau Barbara
Heldmann für das Schreiben des Manuskripts. Herrn Prof. Dr. P. Patzelt danken wir für wertvolle Anregungen im Zusammenhang mit dieser Arbeit. Auch Herrn G. Heinemann und Herrn Dr. W. Schlolaut von der Hessischen Landesanstalt für Tierzucht in Neu-Ulirichstein sei für ihre Hilfe herzlich gedankt.

Summary - Transfer of radioactive cesium from soil to rape plants, rape blossoms and rape honey. Due to the test of atomic weapons and the accident in the nuclear power plant at Chernobyl, the vegetation in Germany has been exposed to cesium contamination in the soil. It was to be expected that activity would migrate from soil to plants and to food products. In this work, the transfer of radioactive cesium from soil to rape plants (Brassica napus var. oleifera), rape blossoms and further to rape honey was investigated. By measuring the gamma activity of cesium using germanium detectors with measuring capacity up to $30 \mathrm{~h}$ per sample (limit of detection about $0.14 \mathrm{~Bq} / \mathrm{kg}$ to $0.19 \mathrm{~Bq} / \mathrm{kg}$ ), we determined a mean transfer factor $\mathrm{f}_{\mathrm{cs}}=$ $0,116 \pm 0,080$ for the system soil-rape plant, $f_{c S}=0.065+0.075$ for the system soil-rape blossom and $F_{c S}=0.098+0.044$ for the system soil-rape honey (plants and honey wet mass, soil dry mass) (Table IV). Additionally, for the transfer of cesium from rape plants to rape honey, $a$ factor of $f_{c s}=$ $2.04 \pm 7.23$ (both wet mass) was determined. Due to some environmental circumstances, which can hardly ever be taken into account, the results obtained sometimes differ considerably. Nevertheless, the mean transfer factors are within the range of values found in literature (Table V).

rape - honey - radioactive contamination - cesium - West Germany 
Résumé - Transfert de césium radioactif du sol au colza, aux fleurs de colza et au miel de colza. A cause d'essais d'armes atomiques et de l'accident de la centrale de Chernobyl, la végétation en Allemagne a été exposée à la contamination par le Césium présent dans le sol. On pouvait s'attendre à ce que la radioactivité migre du sol vers les plantes et se retrouve dans les produits alimentaires. Dans ce travail nous étudions le transfert du Césium radioactif du sol dans le colza (Brassica napus var. oleifera), les fleurs et le miel de colza. En mesurant l'activité gamma du Césium à l'aide de détecteurs au Germanium pendant des périodes de $30 \mathrm{~h}$ par échantillon (limite de détection: $0,14 \mathrm{~Bq} / \mathrm{kg}$ à $0,19 \mathrm{~Bq} / \mathrm{kg}$ environ), nous avons déterminé un facteur moyen de transfert $\mathrm{f}_{\mathrm{Cs}}$ de $0,116 \pm 0,080$ pour le système sol-colza, de $0,065 \pm 0,075$ pour le système sol-fleurs de colza et de $0,098 \pm$ 0,044 pour le système sol-miel de colza (Tableau IV) (poids frais pour les plantes et le miel, poids sec pour le sol). Nous avons en outre déterminé un facteur de transfert $f_{C s}=2,04 \pm 7,25$ pour le système colza-miel de colza (poids frais dans les deux cas). En raison de certains facteurs ambiants, qui peuvent difficilement être pris en considération, les résultats obtenus présentent parfois des variations considérables. Les facteurs moyens de transfert restent néanmoins dans la limite des valeurs trouvées dans la littérature (Tableau V).

colza - miel - contamination radioactive - Césium - Allemagne de l'Ouest

\section{LITERATUR}

Assmann-Wertmüller U. \& Molzahn D. (1989) Die radioaktive Belastung hessischer Honige. Die Biene (1) 6-8
Aurand K., Rühle H. \& Gans I. (1982) Radioökologie und Strahlenschutz. Erich Schmidt Verlag, Berlin

Biesold H. (1981) Der Transfer von Strontium und Caesium über die Wurzeln in die Vegetation. In : Statusbericht über den Transfer von Radionukliden (Der Bundesminister des Innern, Hrsg) 55-72

Biesold H. (1982) Der Transfer radioaktiver Stoffe in landwirstschaftliche Nutzpflanzen. In : Radioökologie und Strahlenschutz (Aurand K. et al., Hrsg). Erich Schmidt Verlag, Berlin, 123132

Der Bundesminister des Inneren, Hrsg (1981) Statusbericht über den Transfer von Radionukliden, 2. Aufl, Bonn

Dustmann J.H. \& van der Ohe W. (1988) Radioaktivitätsmessungen in Honig aus niedersächsischen Trachtgebieten. Nordwestdtsch. Imkerztg. (5) 129-131

Ford B.C., Jester W.A., Griffith S.M., Morse R.A., Zall R.R., Burgett D.M., Bodyfelt F.W. \& Lisk D.J. (1988) Cesium-134 and Cesium-137 in Honey Bees and Cheese Samples Collected in the US after the Chernobyl Accident. Chemosphere 17, 1153-1157

Frederiksson L., Garner R.J. \& Scott Russell R. (1966) Caesium-137. In : Radioactivity and Human Diet (R. Scott Russell, Hrsg) Pergamon Press, 319-352

Heine K. \& Wiechen A. (1978) Bestimmung der Konzentrationsfaktoren von Elementen in der Nahrungskette Boden-Bewuchs-Milch. Milchwissenschaft 33 (4), 230-232

Heine K. \& Wiechen A. (1979) Untersuchungen zum Cs-137-Übergang in der Nahrungskette Boden-Bewuchs-Milch an einem gegebenen Standort. Milchwissenschaft 34 (5), 275-280

Horn H. \& Vorwohl G. (1986) Radioaktive Rückstände im Honig-eine Zwischenbilanz. Allg. dtsch. Imkerztg. 20 (11) 345-348

Jäckel B., Westmeier W. \& Patzelt P. (1987) On the Photopeak Efficiency of Germanium Gamma-Ray Detectors. Nuclear Instruments and Methods in Physics Research A, 261, 543-548

Kaatz H.H. (1986) Heisser Honig, Allg. dtsch. Imkerztg. 20 (7), 222-224

Klepsch A. \& Molzahn D. (1987) Die radioaktive Belastung des Honigs in Hessen nach dem 
Reak-torunglück in Tschernobyl. Die Biene (3) 109-114

Molzahn D. (1987) Marburg nach Tschernobyl. Hitzeroth Verlag, Marburg

Molzahn D., Reinen D., Behr H., Kocksholt P. \& Patzelt P. (1989) Die Belastung von Pilzen mit radioaktivem Caesium - eine Bilanz im hessischen Landkreis Marburg-Biedenkopf im Herbst 1987. Z. Mykol 55 (1), 135-148

Steffens W., Fürh F. \& Mittelstaedt W. (1979/80) Die Aufnahme von Radionukliden aus dem Boden - Bestimmung von Transferfaktoren. Jah- resbericht der Kernforschungsanlage Jülich $\mathrm{GmbH}$

Steffens W., Mittelstaedt W., Führ F., Förstel H. \& Klaes J. (1986) Abschätzung der Aufnahme des abgelagerten ${ }^{137} \mathrm{Cs}$ und ${ }^{90} \mathrm{Sr}$ über die Wurzel, Atomwirtschaft XXXI, 389-392

Westmeier W. (1986) The Fitting of Solid State Detector Spectra. In : Nuclear Instruments and Methods in Physics Research, A, 242, 437-442

Wiesmüller T. (1987) Untersuchungen zur Verteilung der Radioaktivität aus dem Niederschlag von Tschernobyl im Landkreis MarburgBiedenkopf, Diplomarbeit 